Research Article

\title{
Field Equations and Lagrangian for the Kaluza Metric Evaluated with Tensor Algebra Software
}

\author{
L. L. Williams \\ Konfluence Research Institute, 6009 Olympic, Manitou Springs, CO 80829, USA \\ Correspondence should be addressed to L. L. Williams; lance@konfluence.org
}

Received 1 August 2014; Accepted 2 December 2014

Academic Editor: Shinji Tsujikawa

Copyright ( 2015 L. L. Williams. This is an open access article distributed under the Creative Commons Attribution License, which permits unrestricted use, distribution, and reproduction in any medium, provided the original work is properly cited.

\begin{abstract}
This paper calculates the Kaluza field equations with the aid of a computer package for tensor algebra, xAct. The xAct file is provided with this paper. We find that Thiry's field equations are correct, but only under limited circumstances. The full five-dimensional field equations under the cylinder condition are provided here, and we see that most of the other references miss at least some terms from them. We go on to establish the remarkable Kaluza Lagrangian, and verify that the field equations calculated from it match those calculated with xAct, thereby demonstrating self-consistency of these results. Many of these results can be found scattered throughout the literature, and we provide some pointers for historical purposes. But our intent is to provide a definitive exposition of the field equations of the classical, five-dimensional metric ansatz of Kaluza, along with the computer algebra data file to verify them, and then to recover the unique Lagrangian for the theory. In common terms, the Kaluza theory is an " $\omega=0$ " scalar field theory, but with unique electrodynamic couplings.
\end{abstract}

\section{Introduction}

In 1921, Kaluza [1] hypothesized that electrodynamics and general relativity could be unified in terms of general relativity extended to five dimensions. The essence of the Kaluza hypothesis is a specific form for the 5-dimensional (5D) metric tensor; it is an ansatz of the theory. The complete field equations for the Kaluza metric were not discovered for another 25 years, generally attributed to Thiry [2], but recently were shown to be somewhat independently discovered by 4 research groups in the 1940s and 1950s: Jordan and colleagues in Germany, Thiry and colleagues in France, Scherrer working alone in Switzerland, and Brans and Dicke at Princeton [3]. The first English-language translation of the European papers was Thiry [2] in the book by Applequist et al. [4]. The Thiry expressions [2] for the field equations have dominated the literature subsequently for the $5 \mathrm{D}$ theory, and the Brans-Dicke equations have dominated the literature subsequently for general scalar-tensor theories. However, the components of the 5-dimensional Ricci tensor provided by Thiry do not self-consistently produce his expression for the 5D Ricci scalar, so one suspects there is a problem with them.
Moreover, there are diverse expressions in the literature for the associated Kaluza Lagrangian density, and constructions of ever-more-elaborate scalar field Lagrangians [3] have somewhat polluted the minimal scalar field Lagrangian that arises naturally in the Kaluza metric. We would like to base the Lagrangian on the 5D scalar curvature, but there is some question as to the veracity of the scalar curvature calculated by Thiry and others. We shall go on to verify that Thiry's expression for the scalar curvature is indeed correct.

The field equations are difficult to calculate, and neither Thiry nor any of the other references show their calculations. It is difficult to make sense of the differing results and contradictions in the literature. The results presented here are perhaps found in German or French papers from the 1940s and 1950s, as suggested by [3]. But this paper may be the first complete treatment of the Kaluza hypothesis in English. This paper removes any ambiguity by calculating the Kaluza field equations with the aid of a computer software package for tensor algebra, xTensor in the xAct package [5]. The xTensor file is provided online [6]. 


\section{The Metric Ansatz}

In the following, we use Greek indices for spacetime components and the index 5 to indicate the fifth dimension. Roman indices span all 5 dimensions. Tildes are written over $5 \mathrm{D}$ quantities, so that $\widetilde{g}_{a b}$ is the $5 \mathrm{D}$ metric and $g_{\mu \nu}$ is the standard 4D metric. The sign convention for the $4 \mathrm{D}$ metric and curvature tensors is $(-++)$ in the taxonomy of Misner, Thorne, and Wheeler, also known as the Landau and Lifshitz timelike convention.

The Kaluza field equations follow from applying the machinery of general relativity to the ansatz for the 5D metric $\widetilde{g}_{a b}$ :

$$
\tilde{g}_{\mu \nu}=g_{\mu \nu}+\psi A_{\mu} A_{\nu}, \quad \tilde{g}_{5 v}=\psi A_{\nu}, \quad \tilde{g}_{55}=\psi
$$

from which the inverse metric follows:

$$
\tilde{g}^{\mu \nu}=g^{\mu \nu}, \quad \tilde{g}^{5 v}=-A^{\nu}, \quad \tilde{g}^{55}=A^{2}+\frac{1}{\psi} .
$$

The terms quadratic in $A^{\mu}$ were introduced by Klein [7]. The metric (1) is a general decomposition, and the quantities $A^{\mu}$ and $\psi$ are general at this point. But $A^{\mu}$ will be identified as proportional to the electromagnetic vector potential. So the $4 \mathrm{D}$ metric and the electromagnetic vector potential are unified in a 5D metric. The scalar field enters because a $5 \mathrm{D}$ metric will have 15 components. At this point we are only naming components of the $5 \mathrm{D}$ metric. The typical Kaluza treatment identifies $A^{\alpha}$ with the electromagnetic vector potential at the outset, so that a normalizing constant is introduced multiplying $A^{\alpha}$. At this point, we keep the fields unitless and introduce normalization constants at the end.

Along with the form (1), there is another ansatz to the Kaluza theory: the cylinder condition, as Kaluza coined it. It is the assumption that no field depends on the fifth coordinate, $\partial_{5} \widetilde{g}_{a b}=0$. Wesson and colleagues have developed the theory without the constraint of the cylinder condition [8], thereby introducing a huge degree of complexity to the theory. The curvature tensors in this paper were calculated by brute force from the ansatz (1) and its connections under the cylinder condition. The connections are described in the Appendix. We proceed directly to the curvature tensors. All values can be verified in the accompanying xTensor file.

\section{5D Ricci Tensor}

Consider the 5D Ricci tensor $\widetilde{R}_{a b}$, whose components can be summarized in the 3 pieces that distinguish the fifth coordinate: $\widetilde{R}_{\mu \nu}, \widetilde{R}_{\mu 5}, \widetilde{R}_{55}$. To proceed to the 5D Ricci tensor, we evaluate the contracted $5 \mathrm{D}$ Riemann curvature tensor, given by the usual formula:

$$
\widetilde{R}_{a b}=\widetilde{R}_{a c b}^{c}=\partial_{c} \widetilde{\Gamma}_{a b}^{c}-\partial_{b} \widetilde{\Gamma}_{c a}^{c}+\widetilde{\Gamma}_{c d}^{c} \widetilde{\Gamma}_{a b}^{d}-\widetilde{\Gamma}_{b d}^{c} \widetilde{\Gamma}_{a c}^{d}
$$

Since $\widetilde{R}_{555}^{5}=0$ identically,

$$
\begin{aligned}
\widetilde{R}_{55} & =\widetilde{R}_{5 c 5}^{c}=\widetilde{R}_{555}^{5} / \widetilde{R}_{5 v 5}^{v}=\widetilde{R}_{5 v 5}^{v} \\
& =\frac{1}{4} \psi^{2} F^{\alpha \beta} F_{\alpha \beta}+\frac{1}{4 \psi}\left(\partial^{v} \psi\right)\left(\partial_{\nu} \psi\right)-\frac{1}{2} \square \psi,
\end{aligned}
$$

where $\square \equiv \nabla^{\mu} \nabla_{\mu}$ in terms of the $4 \mathrm{D}$ covariant derivative $\nabla_{\mu}$ and where $F_{\alpha \beta} \stackrel{\equiv}{\equiv} \partial_{\alpha} A_{\beta}-\partial_{\beta} A_{\alpha}$. Equation (4) was calculated by hand and used to verify the operation of xTensor at least for this component of the Ricci tensor.

It is customary to set $\psi \equiv \phi^{2}$, in which case a pure divergence is obtained:

$$
\widetilde{R}_{55}=-\phi \square \phi+\frac{1}{4} \phi^{4} F^{\alpha \beta} F_{\alpha \beta}
$$

Since $\widetilde{R}_{\alpha 55}^{5}=0$ identically,

$$
\begin{aligned}
\widetilde{R}_{\alpha 5}= & \widetilde{R}_{\alpha c 5}^{c}=\widetilde{R}_{\alpha 55}^{5}+\widetilde{R}_{\alpha \nu 5}^{\nu}=\widetilde{R}_{\alpha \nu 5}^{\nu} \\
= & \frac{1}{2} \psi g^{\beta \mu} \nabla_{\mu} F_{\alpha \beta}+\frac{1}{4} A_{\alpha} \psi^{2} F^{\mu \nu} F_{\mu \nu}+\frac{3}{4} F_{\alpha \beta} \partial^{\beta} \psi \\
& +\frac{1}{4 \psi} A_{\alpha}\left(\partial^{\nu} \psi\right)\left(\partial_{\nu} \psi\right)-\frac{1}{2} A_{\alpha} \nabla_{\nu}\left(\partial^{\nu} \psi\right) \\
= & \frac{1}{2} \psi g^{\beta \mu} \nabla_{\mu} F_{\alpha \beta}+\frac{3}{4} F_{\alpha \beta} \partial^{\beta} \psi+A_{\alpha} \widetilde{R}_{55} .
\end{aligned}
$$

For the spacetime part of the 5D Ricci tensor, xTensor obtains

$$
\begin{aligned}
\widetilde{R}_{\mu \nu}= & \widetilde{R}_{\mu 5 \nu}^{5}+\widetilde{R}_{\mu \alpha \nu}^{\alpha} \\
= & R_{\mu \nu}-\frac{1}{2 \psi} \nabla_{\nu} \partial_{\mu} \psi+\frac{1}{4 \psi^{2}}\left(\partial_{\mu} \psi\right)\left(\partial_{\nu} \psi\right) \\
& -\frac{1}{2} \psi g^{\alpha \beta} F_{\mu \alpha} F_{\nu \beta}+A_{\mu} A_{\nu} \widetilde{R}_{55} \\
& +A_{\mu}\left(\widetilde{R}_{\nu 5}-A_{\nu} \widetilde{R}_{55}\right)+A_{\nu}\left(\widetilde{R}_{\mu 5}-A_{\mu} \widetilde{R}_{55}\right) \\
= & R_{\mu \nu}-\frac{1}{2} \phi^{2} g^{\alpha \beta} F_{\mu \alpha} F_{\nu \beta}-\frac{1}{\phi} \nabla_{\mu} \nabla_{\nu} \phi \\
& +A_{\mu} A_{\nu} \widetilde{R}_{55}+A_{\mu}\left(\widetilde{R}_{\nu 5}-A_{\nu} \widetilde{R}_{55}\right) \\
& +A_{\nu}\left(\widetilde{R}_{\mu 5}-A_{\mu} \widetilde{R}_{55}\right),
\end{aligned}
$$

where $R_{\mu \nu}$ is the standard 4D Ricci tensor.

For the 5D scalar curvature, xTensor obtains

$$
\begin{aligned}
\widetilde{R} & =g^{\mu \nu} \widetilde{R}_{\mu \nu}-2 A^{\mu} \widetilde{R}_{\mu 5}+\left(A^{2}+\frac{1}{\psi}\right) \widetilde{R}_{55} \\
& =R-\frac{1}{4} \psi F^{\alpha \beta} F_{\alpha \beta}+\frac{1}{2 \psi^{2}}\left(\partial_{\mu} \psi\right)\left(\partial^{\mu} \psi\right)-\frac{1}{\psi} \square \psi \\
& =R-\frac{1}{4} \phi^{2} F^{\alpha \beta} F_{\alpha \beta}-\frac{2}{\phi} \square \phi,
\end{aligned}
$$

where $R$ is the standard 4D scalar curvature.

\section{5D Einstein Tensor}

Let us now assemble the 5D Einstein tensor $\widetilde{G}_{a b} \equiv \widetilde{R}_{a b}-$ $\widetilde{g}_{a b} \widetilde{R} / 2$. We have not yet discussed matter sources, but it is the Einstein tensor that is equated to the stress-energy tensor. 
For the 55-component, xTensor obtains

$$
\begin{aligned}
\widetilde{G}_{55} & =\widetilde{R}_{55}-\frac{1}{2} \psi \widetilde{R} \\
& =\frac{3}{8} \psi^{2} F_{\alpha \beta} F^{\alpha \beta}-\frac{1}{2} \psi R .
\end{aligned}
$$

Remarkably, the equation which would describe $\psi$ in terms of as-yet-unspecified sources is merely algebraic. We will return to this point in the discussion.

For the mixed component of the Einstein tensor, xTensor obtains

$$
\begin{aligned}
\widetilde{G}_{5 v} & =\widetilde{R}_{5 v}-\frac{1}{2} \psi A_{\nu} \widetilde{R} \\
& =A_{\nu} \widetilde{G}_{55}+\frac{1}{2} \psi g^{\alpha \beta} \nabla_{\beta} F_{\nu \alpha}+\frac{3}{4} F_{\nu \alpha} \partial^{\alpha} \psi .
\end{aligned}
$$

We notice that

$$
\widetilde{G}_{5 v}-A_{\nu} \widetilde{G}_{55}=\widetilde{R}_{5 v}-A_{v} \widetilde{R}_{55} .
$$

For the spacetime part of the Einstein tensor, we construct analytically from the foregoing and verify with xTensor:

$$
\begin{aligned}
\widetilde{G}_{\mu \nu}= & \widetilde{R}_{\mu \nu}-\frac{1}{2} \widetilde{g}_{\mu \nu} \widetilde{R} \\
= & R_{\mu \nu}-\frac{1}{2} g_{\mu \nu} R+A_{\mu} A_{\nu} \widetilde{G}_{55} \\
& +A_{\mu}\left(\widetilde{G}_{\nu 5}-A_{\nu} \widetilde{G}_{55}\right)+A_{\nu}\left(\widetilde{G}_{\mu 5}-A_{\mu} \widetilde{G}_{55}\right) \\
& -\frac{1}{2} \psi\left(g^{\alpha \beta} F_{\mu \alpha} F_{\nu \beta}-\frac{1}{4} g_{\mu \nu} F_{\alpha \beta} F^{\alpha \beta}\right) \\
& -\frac{1}{2 \psi}\left(\nabla_{\mu} \partial_{\nu} \psi-g_{\mu \nu} \square \psi\right) \\
& +\frac{1}{4 \psi^{2}}\left[\left(\partial_{\mu} \psi\right)\left(\partial_{\nu} \psi\right)-g_{\mu \nu}\left(\partial_{\alpha} \psi\right)\left(\partial^{\alpha} \psi\right)\right] \\
= & G_{\mu \nu}-\frac{1}{\phi}\left(\nabla_{\mu} \partial_{\nu} \phi-g_{\mu \nu} \square \phi\right) \\
& -\frac{1}{2} \phi^{2}\left(g^{\alpha \beta} F_{\mu \alpha} F_{\nu \beta}-\frac{1}{4} g_{\mu \nu} F_{\alpha \beta} F^{\alpha \beta}\right) \\
& +A_{\mu} A_{\nu} \widetilde{G}_{55}+A_{\mu}\left(\widetilde{G}_{\nu 5}-A_{\nu} \widetilde{G}_{55}\right) \\
& +A_{\nu}\left(\widetilde{G}_{\mu 5}-A_{\mu} \widetilde{G}_{55}\right) .
\end{aligned}
$$

It is striking that (12) produces the exact form of the Maxwell stress-energy tensor.

\section{Comparison with Previous Results}

It is important and illustrative to understand the assumptions and limitations in Thiry's results. Kaluza's original paper contained the result, indeed, the compelling aesthetic feature of the theory, that the gravitational equations with electromagnetic sources could be understood as vacuum equations in a higher-dimensional space. Einstein himself was always aware that the curvature terms of the Einstein equations had a deep and profound beauty that was not reflected in the rather ad hoc stress-energy terms they were equated to, and it was his vision to discover how the matter sources might actually spring from the fields, uniting matter and fields.

So it is sufficient to set the 5D Ricci scalar $\widetilde{R}_{a b}$ to zero and still recover general relativity with electromagnetic sources, and it is a profound result. But such an assumption is not correct for a broader theory which would describe matter. Kaluza originally treated matter sources in the 5D theory and so did not presuppose to set $\widetilde{R}_{a b}=0$. But that assumption appears to have been in much of the subsequent literature.

While Thiry does construct the 5D Einstein tensor $\widetilde{G}_{a b}$, he does so with the implicit assumption that $\widetilde{R}_{a b}$ is zero and so obtains what are truly vacuum equations in both $4 \mathrm{D}$ and $5 \mathrm{D}$ terms. Therefore, Thiry recovers expression (5) for $\widetilde{R}_{55}$, but divided by $\phi^{2}$. Thiry's expression for $\widetilde{R}_{5 \alpha}$ is missing a factor of $\phi$ and missing the term in $\widetilde{R}_{55}$. His expression for $\widetilde{R}_{\mu \nu}$ is similar to (7) but is missing all the terms in $\widetilde{R}_{55}$ and $\widetilde{R}_{\mu 5}$.

As for the 5D Ricci tensor expressions, the Thiry expressions for the $5 \mathrm{D}$ Einstein tensor also drop terms. The expression given for $\widetilde{G}_{5 \alpha}$ is similar to (10) but is missing the term in $\widetilde{G}_{55}$ and is divided by $\phi^{2}$. The expression given for $\widetilde{G}_{\mu \nu}$ is similar to (12) but is missing all the terms in $\widetilde{G}_{55}$ and $\widetilde{G}_{v 5}$.

Interestingly, during his discussion of the 5D Einstein tensor $\widetilde{G}_{a b}$, Thiry fails to write down an expression for $\widetilde{G}_{55}$. He merely refers back to his expression for $\widetilde{R}_{55}$. This has set some subsequent investigations on a wrong path insofar as it is assumed that the equation for the scalar field is given by $\widetilde{R}_{55}=$ 0 in (5). This mischaracterizes the dynamical nature of the scalar field in the $\widetilde{G}_{55}$ equation as shown in (9). Instead, we see that the scalar field is dependent on the 4D scalar curvature $R$ and is not dynamically coupled to the electromagnetic field, as setting $\widetilde{R}_{55}=0$ would lead one to believe.

Another problem with the Thiry expressions for the vacuum equations is that they are not self-consistent. Because of the missing terms, one cannot construct Thiry's expression for $\widetilde{R}$ from Thiry's expressions for $\widetilde{R}_{\mu \nu}, \widetilde{R}_{v 5}, \widetilde{R}_{55}$ and the metric $\tilde{g}_{a b}$. Finally, we note that the beautiful emergence of the electromagnetic stress tensor does not occur in the vacuum $4 \mathrm{D}$ theory.

Among more recent review articles, Overduin and Wesson [8] use the Thiry forms of the field equations. The review article by Coquereaux and Esposito-Farese [9] did provide the correct expression for $\widetilde{G}_{55}$, as well as for the other components of $\widetilde{G}_{a b}$, but was inexplicably missing terms from $\widetilde{R}_{a b}$.

\section{5D Lagrangian}

We now turn to the Lagrange density of the $5 \mathrm{D}$ theory. This will provide an independent check on the field equations just derived and allow us to determine the Lagrangian that corresponds to the field equations. But it will also allow us to 
evaluate the $5 \mathrm{D}$ theory in the context of other scalar field theories. We will find that unnecessary parameterizations of the scalar field have polluted the 5D Lagrangian, which actually needs no kinetic term, contrary to what one commonly sees in the literature, even going back to the work of Jordan in the 1940s [3].

There is of course a freedom in the choice of Lagrangian. The foregoing development was based on the ansatz (1) for the $5 \mathrm{D}$ metric, from which the field equations are obtained through the 5D Einstein equations. But we can also seek the field equations from a corresponding Lagrangian. Just as the Einstein equations were extended directly to five dimensions, we expect that the Hilbert action should also support such an extension. Therefore, one should consider a Lagrangian formed from the 5D scalar curvature.

We can combine (8) with the result, verified by xTensor, that the 5D determinant of $\widetilde{g}_{a b}, \widetilde{g}^{1 / 2}=\psi^{1 / 2} g^{1 / 2}$, where $g^{1 / 2}$ is the determinant of the $4 \mathrm{D}$ metric $g_{\mu \nu}$. We also invoke the cylinder condition, so that the integral $\Delta y$ over the fifth coordinate commutes with the field variables. Let us, therefore, write the 5D Hilbert action and expand it:

$$
\begin{aligned}
\widetilde{S} & =\int \tilde{g}^{1 / 2} \widetilde{R} d^{5} x \\
& =\Delta y \int \psi^{1 / 2} g^{1 / 2} \widetilde{R} d^{4} x \\
& =\Delta y \int g^{1 / 2}\left[\psi^{1 / 2} R-\frac{\psi^{3 / 2}}{4} F^{\alpha \beta} F_{\alpha \beta}-\nabla_{\nu}\left(\frac{\partial^{v} \psi}{\psi^{1 / 2}}\right)\right] d^{4} x \\
& =\Delta y \int g^{1 / 2}\left[\phi R-\frac{1}{4} \phi^{3} F^{\alpha \beta} F_{\alpha \beta}-2 \nabla_{\nu}\left(\partial^{\nu} \phi\right)\right] d^{4} x,
\end{aligned}
$$

where we have set the scalar term to zero because it is a perfect divergence, and as customary the corresponding surface terms are presumed not to contribute at the boundary. As we shall see, the impact of the scalar field in the gravitational equations comes from the term in the scalar curvature.

Let us consider the field equations implied by (13), expanding to explicitly show the inverse metric $g^{\alpha \beta}$, and therefore consider variation of the Lagrangian:

$$
\mathscr{L}=g^{1 / 2}\left[\phi g^{\alpha \beta} R_{\alpha \beta}-\frac{1}{4} \phi^{3} g^{\alpha \mu} g^{\beta \nu} F_{\alpha \beta} F_{\mu \nu}\right] \text {. }
$$

Variation of $\mathscr{L}$ provides, respectively, the equations for $g^{\alpha \beta}$, $A^{\alpha}$, and $\phi$. This would appear to be the unique Lagrangian implied by the Kaluza hypothesis extended to the Hilbert action. Yet this form is rarely written (it is found in the review [9]). Also, (14) would seem to imply that the sign of the fifth dimension in the metric should be timelike so that the proper relative sign between the scalar curvature and electromagnetic invariant is obtained. Some references claim that the sign of the fifth coordinate should be spacelike.

In their review article, Overduin and Wesson [8] repeat the Thiry equations. They also write down a Lagrangian for the $5 \mathrm{D}$ theory, but they do not recover (13). Instead, they have a kinetic-type term for the scalar field proportional to $\partial_{\nu} \phi \partial^{\nu} \phi$. Indeed, scalar field theory does typically have such a quadratic term, and such a term was quickly added to the generalized 5D theory as early as the 1940s [3]. Brans and Dicke [10] adopted a variation of the conventional kinetic term in their Lagrangian, parameterized with the famous $\omega$ coefficient. This $\omega$ family of scalar field extensions to general relativity was further generalized and constrained experimentally by Will [11]. So that now, for any scalar field extension to general relativity, the question arises as to the value of $\omega$. Based largely on the work of Will, it is often repeated that scalar field theories must have $\omega>500$. However, it is also said that general relativity is recovered in the limit as $\omega \rightarrow \infty$, but many counterexamples to that proposition have since been discovered [12], so our understanding of the $\omega$ limits is suspect.

Yet we see that, for the Kaluza Lagrangian (13) or (14), the question of $\omega$ is entirely irrelevant. It can be introduced only to be set later to zero, but that has limited utility. The question arises even why Brans and Dicke felt compelled to introduce an $\omega$ term, given that it is not necessary for a scalar field stress-energy source in the Einstein equations (12). But it was quite the contrary. When Brans and Dicke recover the dynamical scalar field term as in (12), they call it "foreign." They presumably expected a kinetic-type action in their Lagrangian with an adjustable coupling.

It is an expression of the elegance of the Kaluza hypothesis that the scalar field has no tunable coupling parameter. $\omega$ family theories seem extravagant by comparison. Carroll [13] shows how the scalar degree of freedom captured in the $\phi R$ term of the Lagrangian is a basic feature of gravity. For when such a relation as $\phi R$ appears in a Lagrangian, one can recover a standard Lagrangian in $R^{\prime}$ with a coordinate transformation on the metric so that $\phi g_{\mu \nu} \rightarrow g_{\mu \nu}^{\prime}$. But as Carroll shows, this just results in a new term in the Lagrangian that has a kinetic form. So in some sense, a Lagrangian in $\phi R$ without a separate scalar term is the minimal scalar-tensor theory.

Now we proceed to evaluation of the Lagrangian (14). Consider first the field equations for $\phi$ :

$$
\frac{\delta \mathscr{L}}{\delta \phi}=g^{1 / 2}\left(R-\frac{3}{4} \phi^{2} F^{\alpha \beta} F_{\alpha \beta}\right)=0 .
$$

This is identical with (9) for $G_{55}=0$. Note that it is not equivalent to the equation for $\phi$ for $\widetilde{R}_{55}=0$ that is usually used to characterize the scalar field. It seems to constrain the scalar field to the ratio of the scalar curvature to the electromagnetic scalar. The scalar curvature, in the presence of matter, equals the trace of the stress-energy tensor.

Consider now the field equations for $A^{\mu}$. For them, let us use the Euler-Lagrange equations:

$$
\frac{\partial \mathscr{L}}{\partial A_{\mu}}-\nabla_{\nu}\left[\frac{\partial \mathscr{L}}{\partial\left(\nabla_{\nu} A_{\mu}\right)}\right]=0 .
$$

Since Carroll [13] shows the algebra, we can quote his result (1.167) that

$$
\frac{\partial\left(F_{\alpha \beta} F^{\alpha \beta}\right)}{\partial\left(\nabla_{\mu} A_{\nu}\right)}=4 F^{\mu \nu}
$$


Since $\partial \mathscr{L} / \partial A_{\mu}=0$, the field equation for $A^{\mu}$ is

$$
\nabla_{\nu}\left(\phi^{3} F^{\mu \nu}\right)=0
$$

This is identical with (10) for $\widetilde{G}_{\mu 5}=0$ when $\widetilde{G}_{55}=0$. Unlike the scalar-field equation, it is coincidentally the same as its vacuum counterpart in the Ricci tensor, the equation for $\widetilde{R}_{\nu 5}=0$.

Consider now the field equations for the inverse metric $g^{\alpha \beta}$, which is equivalent to finding the equations for the metric. We construct the variation of the Lagrangian in pieces. Carroll has a nice analysis of the scalar field Lagrangian, and we use a couple of his results (4.69 and 4.122) to expedite our variation of $\mathscr{L}$. They are

$$
\begin{gathered}
\delta g^{1 / 2}=-\frac{1}{2} g^{1 / 2} g_{\alpha \beta} \delta g^{\alpha \beta}, \\
g^{\alpha \beta} \delta R_{\alpha \beta}=\nabla_{\nu} \nabla^{\nu}\left(g_{\alpha \beta} \delta g^{\alpha \beta}\right)-\nabla_{\alpha} \nabla_{\beta}\left(\delta g^{\alpha \beta}\right) .
\end{gathered}
$$

As per usual, we shall also neglect perfect divergences of the form

$$
\int g^{1 / 2} \nabla_{\alpha} V^{\alpha} d^{4} x \longrightarrow 0
$$

Varying the first term in the Lagrangian (14) with respect to the metric

$$
\begin{aligned}
\delta \mathscr{L}_{R}= & \phi\left(\delta g^{1 / 2} R+g^{1 / 2} \delta g^{\alpha \beta} R_{\alpha \beta}+g^{1 / 2} g^{\alpha \beta} \delta R_{\alpha \beta}\right) \\
= & \phi g^{1 / 2} \delta g^{\alpha \beta}\left(-\frac{1}{2} g_{\alpha \beta} R+R_{\alpha \beta}\right) \\
& +g^{1 / 2} \phi\left(\nabla_{\nu} \nabla^{\nu} g_{\alpha \beta} \delta g^{\alpha \beta}-\nabla_{\alpha} \nabla_{\beta} \delta g^{\alpha \beta}\right) \\
= & g^{1 / 2} \delta g^{\alpha \beta}\left(\phi G_{\alpha \beta}+g_{\alpha \beta} \nabla^{\nu} \nabla_{\nu} \phi-\nabla_{\alpha} \nabla_{\beta} \phi\right) .
\end{aligned}
$$

The third line is obtained through two successive integrations by parts, neglecting the divergence terms that are understood to be boundary terms as per (21). This is how the dynamical scalar field arises from the minimal Lagrangian.

Varying the second term in the Lagrangian (14) with respect to the metric

$$
\begin{aligned}
\delta \mathscr{L}_{F}= & -\frac{1}{4} \phi^{3} F_{\alpha \beta} F_{\mu \nu} \\
& \times\left(\delta g^{1 / 2} g^{\alpha \mu} g^{\beta \nu}+g^{1 / 2} \delta g^{\alpha \mu} g^{\beta \nu}\right. \\
& \left.+g^{1 / 2} g^{\alpha \mu} \delta g^{\beta \nu}\right) \\
= & -\frac{1}{2} \phi^{3} g^{1 / 2} \delta g^{\alpha \beta}\left(g^{\mu \nu} F_{\alpha \mu} F_{\beta \nu}-\frac{1}{4} g_{\alpha \beta} F^{\mu \nu} F_{\mu \nu}\right) .
\end{aligned}
$$

Now put together the pieces (22) and (23) to obtain the equation for the metric:

$$
\begin{aligned}
\phi G_{\alpha \beta}= & \frac{1}{2} \phi^{3}\left(g^{\mu \nu} F_{\alpha \mu} F_{\beta \nu}-\frac{1}{4} g_{\alpha \beta} F^{\mu \nu} F_{\mu \nu}\right) \\
& +\left(\nabla_{\alpha} \nabla_{\beta} \phi-g_{\alpha \beta} \square \phi\right),
\end{aligned}
$$

which is equivalent to the equation for $\widetilde{G}_{\alpha \beta}=0$ (12) when $\widetilde{G}_{a b}=0$.

So this shows that the unique 5D Hilbert Lagrangian (14) under the Kaluza hypothesis (1) produces the field equations calculated from the connections and curvature tensors. Furthermore, there is no kinetic scalar field term in the Lagrangian; $\omega=0$ in Brans-Dicke terms. The Kaluza hypothesis appears to imply a minimally coupled scalar field with no free parameters.

\section{Appendix}

\section{D Connections}

This appendix gathers the connections calculated from (1), on which the curvature tensors presented above are based. We start with the conventional definition for the affine connection (torsion-free, metric-compatible):

$$
\widetilde{\Gamma}_{b c}^{a}=\frac{1}{2} \widetilde{g}^{a d}\left(\partial_{b} \widetilde{g}_{d c}+\partial_{c} \widetilde{g}_{d b}-\partial_{d} \widetilde{g}_{b c}\right)
$$

All the connections are conveniently written as the six connections that distinguish the fifth coordinate, $\widetilde{\Gamma}_{55}^{5}, \widetilde{\Gamma}_{5 v}^{5}, \widetilde{\Gamma}_{\alpha v}^{5}$, $\widetilde{\Gamma}_{55}^{v}, \widetilde{\Gamma}_{5 \alpha}^{v}, \widetilde{\Gamma}_{\mu \nu}^{\beta}$. We employ the cylinder condition and discard all derivatives with respect to the fifth coordinate. These are straightforward to calculate by hand but are verified with xTensor. They are

$$
\begin{aligned}
& \widetilde{\Gamma}_{55}^{5}=\frac{1}{2} \widetilde{g}^{5 \nu}\left(-\partial_{\nu} \widetilde{g}_{55}\right)=\frac{1}{2} A^{\nu} \partial_{\nu} \psi, \\
& \widetilde{\Gamma}_{5 \nu}^{5}=\frac{1}{2} \widetilde{g}^{5 \alpha}\left(\partial_{\nu} \widetilde{g}_{\alpha 5}-\partial_{\alpha} \widetilde{g}_{5 \nu}\right)+\frac{1}{2} \widetilde{g}^{55} \partial_{\nu} \widetilde{g}_{55} \\
& =\frac{1}{2} A^{\alpha} \psi F_{\alpha \nu}+\frac{1}{2} A^{\alpha} A_{\nu} \partial_{\alpha} \psi+\frac{1}{2 \psi} \partial_{\nu} \psi, \\
& \widetilde{\Gamma}_{\alpha \nu}^{5}=\frac{1}{2} \widetilde{g}^{5 \beta}\left(\partial_{\alpha} \widetilde{g}_{\beta \nu}+\partial_{\nu} \widetilde{g}_{\beta \alpha}-\partial_{\beta} \widetilde{g}_{\alpha \nu}\right) \\
& +\frac{1}{2} \widetilde{g}^{55}\left(\partial_{\alpha} \widetilde{g}_{5 v}+\partial_{\nu} \widetilde{g}_{5 \alpha}\right) \\
& =-A_{\beta} \Gamma_{\alpha \nu}^{\beta}+\frac{1}{2} A^{\beta} A_{\nu} \psi F_{\beta \alpha} \\
& +\frac{1}{2} A_{\alpha} A^{\beta} \psi F_{\beta \nu}+\frac{1}{2}\left(\partial_{\alpha} A_{v}+\partial_{\nu} A_{\alpha}\right) \\
& +\frac{1}{2 \psi}\left(A_{\nu} \partial_{\alpha} \psi+A_{\alpha} \partial_{\nu} \psi\right)+\frac{1}{2} A_{\alpha} A^{\beta} A_{\nu} \partial_{\beta} \psi \\
& \widetilde{\Gamma}_{55}^{\nu}=\frac{1}{2} \widetilde{g}^{\nu \alpha}\left(-\partial_{\alpha} \widetilde{g}_{55}\right)=-\frac{1}{2} g^{\nu \alpha} \partial_{\alpha} \psi, \\
& \widetilde{\Gamma}_{5 \alpha}^{\nu}=\frac{1}{2} \tilde{g}^{\nu \mu}\left(\partial_{\alpha} \tilde{g}_{\mu 5}-\partial_{\mu} \tilde{g}_{5 \alpha}\right)+\frac{1}{2} \tilde{g}^{\nu 5} \partial_{\alpha} \tilde{g}_{55} \\
& =\frac{1}{2} g^{\nu \mu}\left(\psi F_{\alpha \mu}-A_{\alpha} \partial_{\mu} \psi\right),
\end{aligned}
$$




$$
\begin{aligned}
\widetilde{\Gamma}_{\mu \nu}^{\beta}= & \frac{1}{2} \tilde{g}^{\beta \alpha}\left(\partial_{\mu} \tilde{g}_{\alpha \nu}+\partial_{\nu} \tilde{g}_{\alpha \mu}-\partial_{\alpha} \tilde{g}_{\mu \nu}\right) \\
& +\frac{1}{2} \tilde{g}^{\beta 5}\left(\partial_{\mu} \tilde{g}_{5 \nu}+\partial_{\nu} \tilde{g}_{5 \mu}\right) \\
= & \Gamma_{\mu \nu}^{\beta} \\
& +\frac{1}{2} g^{\beta \alpha}\left(A_{\nu} \psi F_{\mu \alpha}+A_{\mu} \psi F_{\nu \alpha}+A_{\mu} A_{\nu} \partial_{\alpha} \psi\right) .
\end{aligned}
$$

There are a couple of convenient identities:

$$
\begin{gathered}
\widetilde{\Gamma}_{55}^{5}+A_{\nu} \widetilde{\Gamma}_{55}^{v}=0, \\
\widetilde{\Gamma}_{v 5}^{v}+\widetilde{\Gamma}_{55}^{5}=\widetilde{\Gamma}_{a 5}^{a}=0 .
\end{gathered}
$$

\section{Conflict of Interests}

The author declares that there is no conflict of interests regarding the publication of this paper.

\section{References}

[1] T. Kaluza, Sitzungsberichte der K. Preussischen Akademie der Wissenschaften zu Berlin, vol. 966, 1921.

[2] M. Y. Thiry, "Geometry-the equations of Kaluza's unified theory," Comptes Rendus de l'Académie des Sciences, vol. 226, p. 216, 1948.

[3] H. Goenner, "Some remarks on the genesis of scalar-tensor theories," General Relativity and Gravitation, vol. 44, no. 8, pp. 2077-2097, 2012.

[4] T. Applequist, A. Chodos, and P. G. O. Freund, Modern KaluzaKlein Theories, Addison-Wesley, New York, NY, USA, 1987.

[5] xTensor, in the xAct package, http://www.xact.es/.

[6] "xTensor computer tensor algebra file," http://www.konfluence.org/.

[7] O. Klein, "Quantentheorie und fünfdimensionale Relativitätstheorie," Zeitschrift für Physik, vol. 37, no. 12, pp. 895-906, 1926.

[8] J. M. Overduin and P. S. Wesson, "Kaluza-Klein gravity," Physics Report, vol. 283, no. 5-6, pp. 303-378, 1997.

[9] R. Coquereaux and G. Esposito-Farese, "The theory of Kaluza-Klein-Jordan-Thiry revisted," Annales de l'Institut Henri Poincare Physique Theorique, vol. 52, p. 113, 1990.

[10] C. Brans and R. H. Dicke, "Mach's principle and a relativistic theory of gravitation," Physical Review, vol. 124, no. 3, pp. 925935, 1961.

[11] C. Will, Theory and Experiment in Gravitational Physics, revised edition, Cambridge University Press, Cambridge, Mass, USA, 1993.

[12] V. Faraoni, "Illusions of general relativity in Brans-Dicke gravity," Physical Review D, vol. 59, no. 8, 1999.

[13] S. Carroll, Spacetime and Geometry, section 4.8, Addison Wesley, San Francisco, Calif, USA, 2004. 

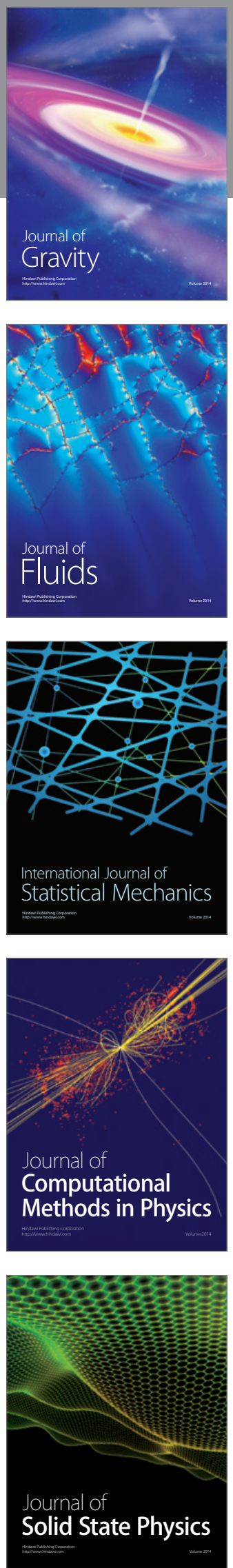

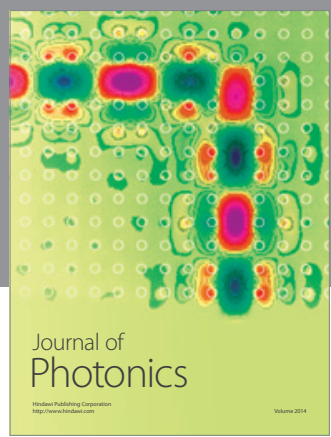

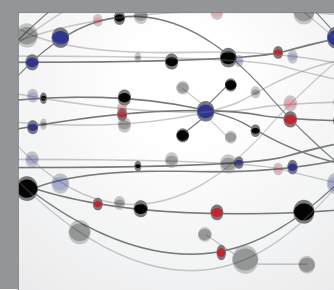

The Scientific World Journal

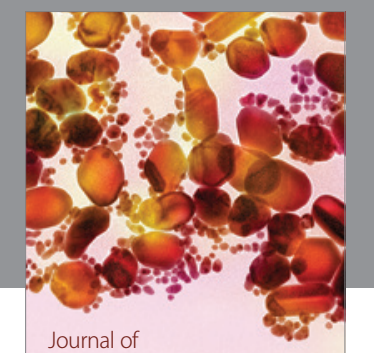

Soft Matter
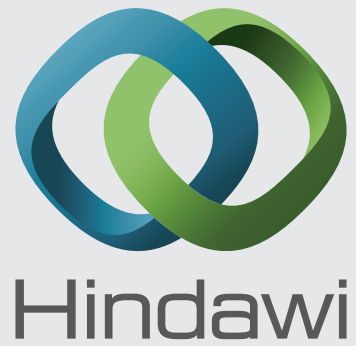

Submit your manuscripts at

http://www.hindawi.com
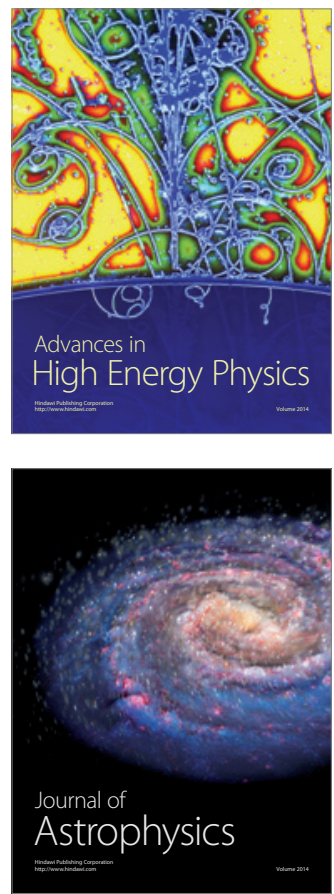
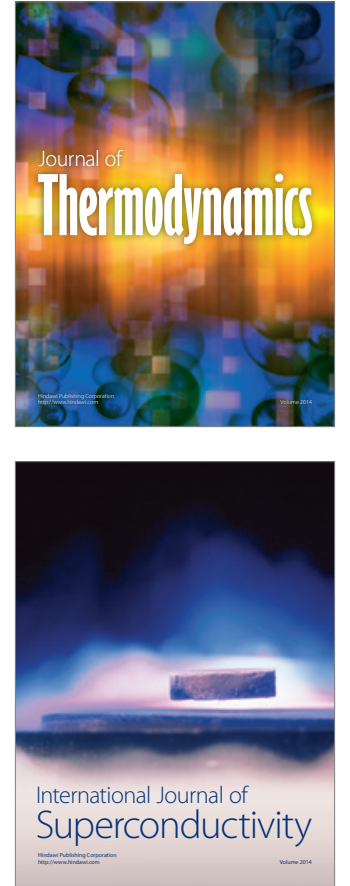
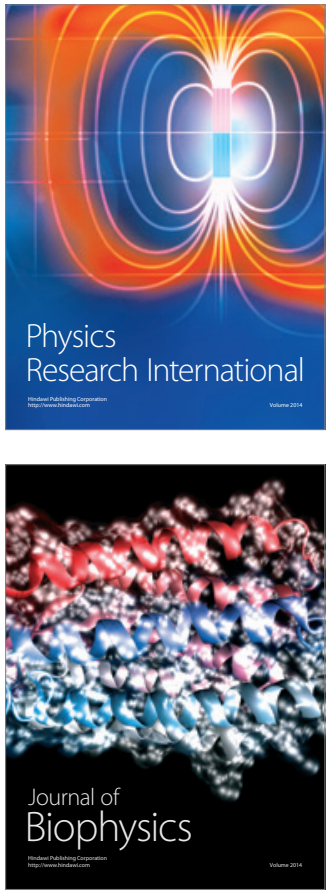
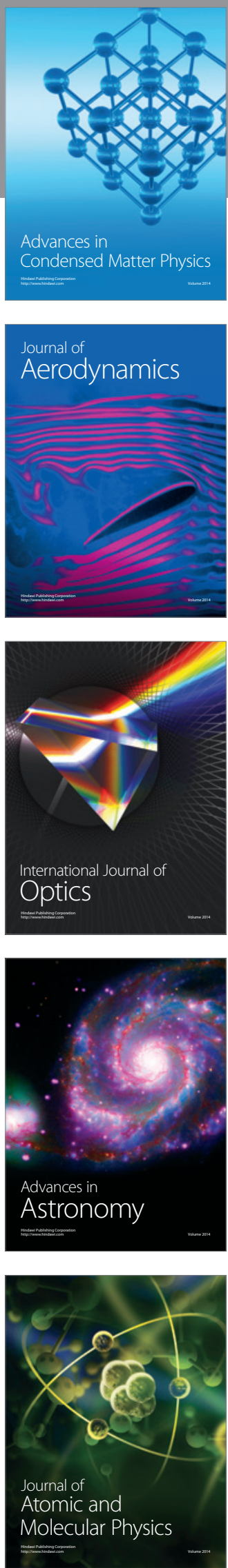